\title{
The Dawn (Diabetes: Attitudes, Wishes and Needs) of Type 2 Diabetes Mellitus in Trinidad, A Small Middle Income Developing Country
}

\author{
Kameel Mungrue*, Lilly Paul, Brandon Ramdass and Koreen Peter \\ Department of Paraclinical Sciences, University of the West Indies, Trinidad
}

Submission: Febrvary 8, 2017; Published: May 04, 2017

*Corresponding author: Kameel Mungrue, Department of Paraclinical Sciences, University of the West Indies, Trinidad, Tel: 8686452018 ; Email: kameel.mungrue@sta.uwi.edu

\begin{abstract}
Objective: To investigate how people with T2DM perceive diabetes care and to establish a person-centred model that emphasizes the individual needs of the patient, in the context of current chronic care, education and psychological support and self-management.

Design and Methods: This was a cross-sectional observational study conducted at the Arima and Sangre Grande Health Facilities. Questionnaires, which were constructed using internationally validated questionnaires, were administered by interview among adult patients with diabetes.

Results: Of the 301 participants entered into the study the age at diagnosis gradually decreased as well as the highest proportion of cases shifted from 69 years to the 49-58 and 59-68 age groups. 169 people ranked between 0-3 on the PAID-5 scale, indicating that 56.1\% of the sample experienced no real emotional distress or depression as a result of their diabetic condition. At one site a total of $46.9 \%$ of people felt that their care was well organized most of the time to all of the time while at the other site, $63.5 \%$ of people felt that their health care was well organized most of the time to all of the time. Furthermore, patient adherence to diet and exercise interventions were poor, compared with the use of medications. Of the patients interviewed, 58.5\% had never taken part in any diabetes educational programme.
\end{abstract}

Conclusion: Psychosocial problems are prevalent in our setting. Issues with patient satisfaction, compliance and education need to be addressed to holistically meet the needs of the patient.

Abbreviations: WHO: World Health Organisation; LMICs: Low and Middle-Income Countries; TTO: Trinidad and Tobago; DAWN: Diabetes Attitudes Wishes and Needs; PWD: People With Diabetes; RHA: Region Health Authorities; PHCF: Primary Health Care Facilities; BMI: Body Mass Index

\section{Introduction}

According to the World Health Organisation (WHO), the number of adults living with type 2 diabetes (T2DM) has almost quadrupled since 1980 to 422 million in 2016, accounting for 1.5 million deaths in 2012 [1]. The new report calls upon governments to ensure that people are able to make healthy choices and that health systems are able to diagnose, treat and care for people with diabetes. Rapid economic development accompanied by environmental, social and behavioural change occurred in many low and middle-income countries (LMICs) since the 1980s. In fact the epidemiological transition characterized by falling infectious disease rates with increasing rates in noncommunicable diseases occurred in Trinidad and Tobago (TTO) as far back as 1947 [2]. The prevalence of T2DM in TTO range from 78-112 per 1000 population, The Ministry of Health estimates the prevalence of T2DM at 111 per 1000 population or 1 in 8 adults [3]. The economic burden -for the supply of medical treatments and therapeutic interventions, as well as delivering optimal healthcare services- impacts adversely on an already overburdened health system. Barcelo and collegues placed TTO in group 1 countries with an expenditure for T2DM per capita gross national product (GNP) $>6000$ (USD) and an average cost per person of 577(USD) [4].

Despite the provision of dedicated services such as chronic disease clinics most people with T2DM do notachieve target blood glucose levels by international guidelines $[5,6]$. This implies that optimal management of T2DM go beyond clinical domains. In 


\section{Current Research in Diabetes \& Obesity Journal}

2001, the global Diabetes, Attitudes, Wishes and Needs (DAWN) study provided insights into the psychosocial challenges faced by people with diabetes [7]. The study showed that psychosocial problems can be barriers to achieving adequate glycaemic control [8]. Exploiting the increasing popularity of social media and the opportunities they provide for social support, in 2011, international experts and organisations, including the International Diabetes Federation, the International Alliance of Patients' Organizations (IAPO) and the Steno Diabetes Centre, in collaboration with Novo Nordisk implemented the DAWN (2) study [9]. The DAWN 2 study builds on the experience gained in the behavioral and psychological sciences since the first DAWN (1) study in 2001 [10]. It seeks to bridge gaps highlighted by the DAWN(1) study and to bring about improvements in all strata of health care including the individual, health care organizations, communities and government policy makers [11]. A review of the literature found no published data for the Caribbean.Thus, this is the first study of its kind to explore the psychosocial challenges among patients with T2DM, in Trinidad. Specifically, the study will assess the ability of people with diabetes (PWD) to self-manage their condition and to determine levels of psychosocial distress.

The aim of this study is to replicate DAWN(2) among people with T2DM with particular emphasis on the perception of diabetes care and to establish a person-centered model that emphasizes the needs of the individual in the context of current chronic care, self-management, education and psychological support. In addition the study also aims to identify the shortfalls of the current health system in Trinidad and Tobago in regard to care delivered to patients with T2DM. Further to identify causes of patient non-compliance, avenues facilitate dialogue and collaboration to strengthen patient involvement and improve self-management and psychosocial support in diabetes care and to establish a validated survey system for assessing and benchmarking psychosocial and educational aspects of diabetes care delivery across the Caribbean.

\section{Methods}

We used a prevalence study design. The population consisted of all adult patients ( $>18$ years) with T2DM seeking care at primary health care facilities (PHCF). We used a multistage sampling process. Trinidad is divided into four region health authorities (RHA) for the purposes of delivering health care. In the first stage we randomly selected two of the four RHA, i.e. RHA- 1 and RHA-2. RHA- 1 has 15 PHCF and the RHA-2 has 12 PHCF. In the second stage we randomly selected one PHCF from each RHA. We considered these two PHCF as clusters therefore all clients attending these PHCF were eligible for entry into the study. We choose one facility to avoid varying clinical practices and to control simultaneously for the possible confounding effects of the different variables.

All patients with a physician diagnosis of T2DM or a fasting blood sugar of $\geq 126 \mathrm{mg} / \mathrm{dL}$, or a random blood sugar of $\geq 200 \mathrm{mg} / \mathrm{dL}$ or an $\mathrm{HbA} 1 \mathrm{c} 7 \%$ were eligible for entry into the study. All patients with gestational diabetes or type 1 diabetes were excluded from the study. All data were collected using a specifically designed data collection instrument, which include demographic data, i.e. age, gender, ethnicity, body mass index (BMI), education level and treatment together with items from the DAWN (2) questionnaire. In addition, we also used the Problem Areas in Diabetes Questionnaire, PAID (5). The PAID measure of diabetes related emotional distress correlates with measures of related concepts such as depression, social support, health beliefs, and coping style, as well as predicted future blood glucose control of the patient. The diagnostic accuracy of the scale is acceptable, achieving a sensitivity $95 \%$ and a specificity rate of $89 \%$. A major strength of the PAID-5 is that it takes less than 1 min to complete; yet it has comparable 'diagnostic' performance of the four-item Diabetes Distress Scale [12]. Each question has five possible answers with a value from 0 to 4 , with 0 representing "no problem" and 4 "a serious problem". The scores are added up and multiplied by 1.25 , generating a total score between $0-100$. Patients scoring 40 or higher may be at the level of "emotional burnout" and warrant special attention.

The Summary of Diabetes Self-Care Activities (SDSCA) measure is a brief self-report instrument for measuring levels of self-management across different components of the diabetes regimen [12]. The SDSCA assesses levels of self-care and not adherence or compliance to a prescribed regimen because of the difficulties associated with identifying, for a given patient, a specific unchanging standard against which behavior should be compared [13-15]. Diabetes self-care includes a range of activities (e.g., self-monitoring of blood glucose, eating a lowsaturated-fat diet, and checking one's feet) and it is now well established that these different components do not correlate highly $[14,16,17]$. Because self-care is multidimensional, it is necessary to assess each component separately rather than to combine scores across components [13]. Hence we focused on the general diet only exploring the following 5 items (followed your eating plan, fruits and vegetables, high fat foods, carbohydrates, followed a healthy eating plan). Participants could respond: not at all during the past 7 days, or to each of 1-7 days. Scores ranged from 1 (no weekly participation in diabetes self-care activity) to 8 (participation in diabetes self-care activities every day during the past week). Item scores were averaged resulting in an overall score for each item of the self-care activity. A sample size of 300 was calculated if participants completed the entire the questionnaire the maximum attainable average is 448 , i.e. if all 300 participants performed the activity every day i.e. the desired outcome.

The final component of the study examined perceptions of diabetic care. This was assessed using the Patient Assessment of Chronic Illness Care (PACIC) which consisted of twenty (20) items. Each item was scored 1-5 with the exception of items 1 and 9 , which were scored 0 and 1 , in our setting. Hence our mean score varied between 0.9-4.8 in comparison to PACIC scores of 1-5. All questionnaires were pretested and subsequently adapted to a Trinidadian population. All data were stored, retrieved and 


\section{Current Research in Diabetes \& Obesity Journal}

analyzed using SPSS version 22 . A value $\mathrm{p} \leq 0.05$ was considered significant. Ethical approval for this study was obtained from the Ethics Committee of the University West Indies St.

\section{Results}

There were 320 eligible participants who met the criteria for entry at the two primary care clusters selected. The sample consisted of 194 (64.5\%) participants from one cluster and $107(35.5 \%)$ from the other, thus 301 participants entered the study and 19 refused, giving a non-response rate of 5.9\%. All 301 participants fulfilled the entry criteria were entered into the study and were available for analysis. The mean age was 60.78 years ( $\mathrm{SD} \pm 11.5)$ with an interquartile range of 51-70 years, table1. There are two major diaspora in Trinidad, Africans and South East Asians (SEA) both representing approximately 35\% of the population respectively. However T2DM is higher among SEA compared to Africans [3]. Notwithstanding, $42 \%$ of our sample consisted of participants of Africans and 33.2\% were SEA. There were more females $(179,59.5 \%)$ than males 122 (40.5\%), f:m 1.5:1.

Participants were asked about their living situation. The majority reported that they lived with a spouse/partner or children $(151,34.6 \%)$, and only 53(12.2\%) participants lived alone. Of those who lived with children, 96(22\%) lived with a child $>18$ years old while only $52(1.9 \%)$ lived with a child $<18$ years. The majority of participants in the study were retired $(132,42 \%)$, only $82(26.1 \%)$ were currently employed full time and $19(6.1 \%)$ reported that they were unable to work due either to their T2DM directly or to complications arising. In Trinidad family support for patients with T2DM is high, as 195(64.8\%) participants said their family was 'very supportive'. Further 98(32.6\%) participants reported that their health care team was 'somewhat supportive' and $176(58.5 \%)$ reported 'very supportive'.

Participants were asked about their living situation. The majority reported that they lived with a spouse/partner or children $(151,34.6 \%)$, and only $53(12.2 \%)$ participants lived alone. Of those who lived with children, $96(22 \%)$ lived with a child $>18$ years old while only $52(1.9 \%)$ lived with a child $<18$ years. The majority of participants in the study were retired $(132,42 \%)$, only $82(26.1 \%)$ were currently employed full time and $19(6.1 \%)$ reported that they were unable to work due either to their T2DM directly or to complications arising. In Trinidad family support for patients with T2DM is high, as 195(64.8\%) participants said their family was 'very supportive'. Further 98(32.6\%) participants reported that their healthcare team was 'somewhat supportive' and 176(58.5\%) reported 'very supportive'.

Most participants $(285,94.7 \%)$ did not experience discrimination and stigma even along ethnic lines. Of the small number of participants $16(5.3 \%)$ who felt they were discriminated because of their T2DM, identified co-worker harassment and judgmental behavior or failure to be hired as main sources of discrimination.Prospective and cross-sectional studies consistently point to the fact that diabetic patients are more likely to develop micro- as well as macro-vascular complications [18-20] which reduce the quality of life of patients, incur heavy burdens to the health care system, and increase diabetic mortality [21-23]. About 50\% of the subjects of UKPDS had substantial macro- or micro-vascular abnormalities at the time of T2DM diagnosis [24]. We therefore inquired about the common macrovascular (coronary artery disease, stroke and peripheral vascular disease) and micromascular (retinopathy, nephropathy and neuropathy) complications patients were currently experiencing. More than half the number of participants in the study, 167 (55.5\%) had either a mico-or macrovascular complication. The most common complication reported was retinopathy $(79,26.3 \%)$; other complications included neuropathy $(42,14 \%)$ and coronary heart disease (39, $13 \%)$. Further sleeping problems $(54,18 \%)$ were more common than sexual dysfunction $(5,1.7 \%)$. Hypertension was the most common comorbidity $(46,15.3 \%)$.

Since retinopathy, was the most common complication, a stepwise logistic regression was performed to determine the risk factors most likely associated retinopathy. Two significant $(\mathrm{p} \leq 0$. 05 ), risk factors were identified age and duration of diabetes. Older participants were twice as likely to develop retinopathy (OR 2.23, 95\%CI 1.05-4.96, p=0.038), while the longer the duration of T2DM, the greater the risk of developing retinopathy (OR 2.89, 95\%CI 1.68,4.86, p=0.001). Current guidelines from the American Diabetes Association/European Association for the Study of Diabetes (ADA/EASD) and the American Association of Clinical Endocrinologists/American College of Endocrinology (AACE/ACE) recommend early initiation of metformin as a firstline drug for monotherapy and combination therapy for patients with T2DM $[25,26]$. Oral antidiabetic agents (OAA) were the most commonly prescribed and used treatment (242, 80.4\%) of which $(198,65.8 \%)$ was prescribed Metformin. Metformin's first-line position was strengthened by the United Kingdom Prospective Diabetes Study (UKPDS) observation that the metformin-treated group had risk reductions of $32 \%(\mathrm{p}=0.002)$ for any diabetes-related endpoint, $42 \%$ for diabetes-related death $(\mathrm{p}=0.017)$, and $36 \%$ for all-cause mortality $(\mathrm{p}=0.011)$ compared with the control group [27]. Insulin therapy was administered to $126(42 \%)$ participants. Most participants (190, $63.1 \%$ ) reported that they received all their medication free, but were required to wait for long periods before receiving them. Of the $111(36.9 \%)$ who made out of pocket payments for some of their medications, $(87,78.4 \%)$ indicated its negative impact on their financial resources.

Living with T2DM requires that patients develop a range of competencies that allow them to take greater control over the treatment of their disease. This requires educational programs with the aim of enhancing active involvement of patients so that they become partners in their health care process that promotes health and achieves better outcomes. Although programs are 


\section{Current Research in Diabetes \& Obesity Journal}

available 176(58.5\%) participants never participated. Of the $126(41 . \%)$ who did participate, 18(6.0\%) found it not helpful, $41(13.6 \%)$ reported it was somewhat helpful and $80(26.6 \%)$ found it very helpful. On the other hand $114(21.7 \%)$ participants relied on doctors/nurses to fulfill this need, and in our setting family and friends $(101,19.2 \%)$ were also an important source of information.

Participants were asked about the impact of diabetes on physical health; more participants reported that it negatively affected their physical health than those who said it did not $(112,37.2 \%)$. Also, 122 people stated that diabetes did not affect their financial situation adversely. Other areas explored were the relationships with family and friends in which 65 participants reported that the relationship improved, (171) reported that there was no change, while. $138(50 \%)$ reported that leisure activities was adversely affected.

Only 105(34.9\%) participants reported that they followed a healthy eating plan daily for the last week, while $42(14.0 \%)$ participants had not followed a healthy eating plan at all. Furthermore, 95(31.6\%) participants did not engage in physical activity for 30 minutes or more in any of the previous 7 days. however, a high proportion of participants $(212,70.4 \%)$ reported that they complied with the doctor's instruction and took their diabetes medication exactly as prescribed. More than half of the sample also checked their feet daily $(166,55.1 \%)$.

The WHO-Five Well-being Index (WHO-5) was used to evaluate quality of life. The majority of participants $(153,50.8 \%$, score $>71$ ) felt contented with their quality of life, despite having T2DM, 5(1.7\%) participants scored $\leq 28$, which indicates likely depression while 45(15\%) participants scored between 29-49 indicating that they are at risk for depression.

When the PAID questionnaire was administered two participants failed to answer all the questions and were therefore unable to be ranked. We found 169 people ranked between $0-30$ on the PAID5 scale, indicating that $56.1 \%$ of the sample experienced no real emotional distress or depression as a result of their diabetic condition. There were $44(14.6 \%)$ people ranked between 40 and 70 . There were 86 participants ranked between 80 and 100 , indicating the possibility of diabetes related distress, which warrants further assessment.

Using (SDSCA 6) we found that overall performance on each item as well as an aggregate of all items was poor. Although 105(34.9\%) participants followed a healthy eating plan daily for sevens only $42(14 \%)$ did not reduce their fat intake, table 2 . The optimal performance for this category is 478 the attained performance was only $72.8(15.2 \%)$. Fruit and vegetable (F \& V) consumption was also consistently poor 22 participants reported that they did not consume F \& V over the past 7 days.

In regard to examination of the feet, 188 people (62.5\%) did not have their feet examined by a healthcare professional. In addition $215(71.4 \%)$ participants mentioned that no one enquired if they had felt worried or sad. Lastly, 193(64.1\%) of people said that no healthcare professional ever asked about the types of food they ate. In the previous 12 months, 192(63.8\%) participants reported that their HbA1c was not measured while 91(30.2\%) indicated that were not sure if it had been measured.

We report a mean score of 1.5 for the PACIC arm of the study. There was a significant difference $(\mathrm{p} \leq 0.05)$ between the two centers studied, more patients (63\%) in RHA-1 were satisfied that their health care needs i.e. "well taken care of ", "most of the time", compared to RHA-2 (47\%). The main complaint was the long waiting times experienced prior to their consultation. Other complaints included no written list of instructions or treatment plan were ever given $(223,74 \%)$ to follow, and $231(76 \%)$ also indicated that there was no discussions on their current treatment plan, nor were they asked about goals or targets. The majority of participants $(272,90 \%)$ reported that they were not encouraged to attend specific educational interventions. Further $249(83 \%)$ of participants reported that their values, beliefs and traditions were never solicited in regards to their treatment. Few participants $45(15 \%)$ were referred to a dietician and $186(61.8 \%)$ said that they were never asked about problems they were experiencing with their medication. Apart from the contact in the clinic there was no other contact between the participant and their doctor/nurse.

\section{Discussion}

An important finding of the study was a steady increase in the number of persons diagnosed and treated with T2DM between the 1990's and the first decade of the 21st century and the vulnerability of adolescents and young adults. At the two PHCF studied, for the first time they admitted patients in the age group 19-28, a disease once thought to be a metabolic disorder exclusively of adulthood [28]. This trend in the occurrence of T2DM is not unique to our setting. For example, in Japan $80 \%$ of all new cases of diabetes in children and adolescents were diagnosed as T2DM [29]. Similar patterns have been reported in Taiwan [30], and to a less extent in Europe, U.K. and the U.S.A. $[31,32]$. T2DM is progressive, and one main factor responsible for this is a continued decline in $\beta$-cell function [33]. In addition overt T2DM does not occur until the $\beta$-cell fails to compensate appropriately to the peripheral insulin resistance state. The ability of the $\beta$-cell to secrete sufficient insulin to adequately respond to the peripheral insulin resistance state depends on multiple factors, including $\beta$-cell mass [34] and secretory capacity [35], which are influenced by genetic and environmental factors [36]. While little can be done in regard to genetic factor, much can be achieved by tackling environmental factors. Improving the physical environment such asincreasing availability of healthy food choices and opportunities for physical activity together with a structured lifestyle-change program is vital to reducing T2DM among people at high risk. Both randomized clinical trials and real-world implementation studies have proven that structured lifestyle-change programs can help prevent or delay T2DM by $60 \%$ in people with prediabetes. 
High quality chronic illness care can be defined as having a PACIC score between 4 and 5 [15], we report for the first time a score of 1.5. PACIC consist of five (5) subscales; items 1-3 are Patient Activation which assess actions that solicit patient input and involvement in decision making; items 4-6 are Delivery System Design/Decision Support which evaluate actions that organize care and provide information to patients to enhance their understanding of care; items 7-11 are Goal Setting, these items evaluate the acquisition of information for and the setting of specific, collaborative goals; items 12-15 are Problem solving/ Contextual counseling which consider potential barriers and the individual's social and cultural environment in making treatment plans; and items 16-20 are Follow Up/Coordination which evaluates the arrangement of care that extends and reinforces office based treatment and making proactive contact with patients to assess progress and coordinate care. Houle et al in 2012 reported a score of 2.8 while scores of 3.1 and 3.2 have been reported from studies done in the USA [37]. In the developed world most people report a PACIC score ranging between 3.1-3.2 [38-46]. This finding therefore suggests a lower standard of care compared with the developed world. Some contributing factors included the long waiting times, being unable to see the same doctor at return visits, lack of empathy from members of the healthcare team and the consultation was impersonal and was mainly used to repeat prescriptions. Further there was a lack of diabetes educational programs and support groups. Emotional distress due to diabetes was assessed using Problem Areas in Diabetes Scale (PAID)-5, which has been validated as a reliable tool to assess diabetes emotional distress [47]. The majority of participants viewed T2DM as a condition, which they were able to cope with, and showed little to no signs of worry or anxiety; this finding is consistent with other [48].

The quality of life for diabetic patients was assessed using the WHO5 Well Being Index. We found 83\% of participants scored greater than 50 indicating that these individuals were generally in good-high mood. Similar findings have been reported in the literature [49]. Further $17 \%$ of participants had scores below 50 indicative of a low mood but only $2 \%$ had scores below 28 which represents a high risk group for clinical depression [50]. Although the study was not intended to measure the prevalence of depression, the finding was markedly different from the 17\% reported by Frederick [51]. Depression is common among people with diabetes [52], especially among those with diabetic complications [53]. Depression has been associated with poor adherence to medication [54], poor glycemic control [55], as well as with the development of diabetic complications [56] and increased mortality [57]. Several studies focusing on the prevalence of depression in people with diabetes have been done, showing different depression rates [58,59].

Although participants reported that they were compliant with prescribed medications and checking their feet as recommended, they were less compliant with following a healthy diet, monitoring their blood sugar and getting the recommended 30 minutes or more of physical activity. Nicolucci et al. [11] in a previous DAWN 2 study found that persons were more likely to follow self-care advice about medication and diet and less likely to follow blood glucose testing, physical activity and foot examination guidelines. In Trinidad and Tobago, there is a free drug distribution program called the Chronic Disease Assistance Program (CDAP), through which medications such as insulin and oral antiglycimic agents are available to all citizens. (5) This may in part contribute to the high compliance in our setting, since Nicolucci et al. [11] reported that $25 \%$ of respondents in the initial DAWN2 study had difficulty paying for their diabetes medication, which was contributing to noncompliance. On the other hand blood glucose monitoring was poor because participants are required to purchase test strips. Similarly only $50 \%$ of participants reported that their $\mathrm{HbA} 1 \mathrm{c}$ was measured over the past year.

Diabetic retinopathy was the main complication among participants. Using binary logistic regression two significant risk factors were identified i.e. duration of diabetes (having been diagnosed with diabetes for more than 10 years) and age over 50. The main implication of this finding is the demand for ophthalmologists of which there are few employed in the public health sector. Retinopathy patients require other types of medication, and this adds to the burden that diabetes places on the health sector.

The sample of 301 persons was restricted to only two PHCF and therefore suffers from generalizability. The study cannot account for undiagnosed T2DM and therefore underestimates disease burden, mainly due to the study design. Nevertheless, it suggests a potentially meaningful burden of disease. Thus, we recommend a nationally representative sample of adolescents and young adults to address the emerging challenge. The questionnaire length affected cooperation rates and participants had to be encouraged to participate. However when this information is combined through a mixed method approach, robust results can be obtained, which are both comprehensive and quantifiable, adding an invaluable perspective to the analysis of the situation.

\section{Conclusion}

In conclusion the study provides evidence of the unmet needs of the patient with T2DM. In a changing world and more so in the developing world provision of service has to go beyond the provision of pharmaceutical agents if we are to favorable impact on the outcomes of chronic noncommunicable diseases such as T2DM.

\section{References}

1. WHO (2016) Global Report on Diabetes. World Health Organizaiton, Geneva, Switzerland.

2. Mungrue K (2012) The changing face of death in Trinidad and Tobago, before and after independence. West Indian Med J 61(4): 452-459. 


\section{Current Research in Diabetes \& Obesity Journal}

3. World diabetics day (2010) Ministry of Health Government of the Republic of Trinidad and Tobago.

4. Barcelo A, Aedo C, Rajpathak S, Robles S (2003) The cost of diabetes in Latin America and the Caribbean. Bull World Health Organ 81(1): 19-27.

5. Piya MK, Tahrani AA, Barnett AH (2010) Emerging treatment options for Type 2 Diabetes. Br J Clin Pharmacol 70(5): 631-644.

6. Nam S, Chesla C, Stotts NA, Kroon L, Janson SL (2011) Barriers to Diabetes management: patient and provider factors. Diabetes Res Clin Pract 93(1): 1-9.

7. Skovlund SE, Peyrot M (2005) The Diabetes Attitudes, Wishes, and Needs (DAWN) program: A New Approach to Improving Outcomes in Diabetes Care. Diabet Spectr 18(3): 136-142.

8. Peyrot M, Rubin RR, Lauritzen T, Snoek FJ, Matthews DR, et al. (2005) Psychosocial problems and barriers to improved diabetes management: Results of the Cross-National Diabetes Attitudes, Wishes and Needs (DAWN) Study. Diabet Med 22(10): 1379-1385.

9. Novo Nordisk (2014) DAWN STUDY: Diabetes Attitudes, Wishes and Needs.

10. Peyrot M, Burns KK, Davies M, Fobes A, Hermanns N, et al. (2013) Diabetes Attitudes, Wishes and Needs 2 (DAWN2): A multinational, multi-stakeholder study of psychosocial issues in diabetes and personcentred diabetes care. Diabetes Res Clin Pract 99(2): 174-184.

11. Zandbelt N, Eldrup E, Gagliardino JJ, et al. (2008) Well-being and glycemic control in diabetes patients: preliminary data from the crossnational DAWN Monitoring of Individual Needs in Diabetes(MIND) study. Diabetes 57 (Suppl 1).

12. Toobert DJ, Glasgow RE (1994) Assessing diabetes self-management: the summary of diabetes self-care activities questionnaire. In Handbook of Psychology and Diabetes. Bradley C, (Eds) Chur, Switzerland, Harwood Academic pp. 351-375.

13. Johnson SB (1992) Methodological issues in diabetes research Diabetes Care 15(11): 1658-1667.

14. Glasgow RE, Anderson RM (1999) In diabetes care, moving from compliance to adherence is not enough: something entirely different is needed. Diabetes Care 22(12): 2090-2092.

15. Glasgow RE, Wilson W, McCaul KD (1985) Regimen adherence: a problematic construct in diabetes research. Diabetes Care 8(3): 300301

16. Rubin RR, Peyrot M (1992) Psychosocial problems and interventions in diabetes. Diabetes Care 15(11): 1640-1657.

17. Orme CM, Binik YM (1989) Consistency of adherence across regimen demands. Health Psychol 8(1): 27-43.

18. Lee ET, Keen H, Bennett PH, Fuller JH, Lu M (2001) Follow-up of the WHO Multinational Study of Vascular Disease in Diabetes: general description and morbidity. Diabetologia 44(2): S3-13.

19. Le Roith D, Fonseca V, Vinik A (2005) Metabolic memory in diabetesfocus on insulin. Diabetes Metab Res Rev 22(2): 85-90.

20. Turner RC, Holman RR (1995) Lessons from UK prospective diabetes study. Diabetes Res Clin Pract 28(Suppl): S151-S157.

21. Wang W, Fu CW, Pan CY, Chen W, Zhan S, et al. (2009) How do type 2 diabetes mellitus-related chronic complications impact direct medical cost in four major cities of urban China? Value Health 12(6): 923-929.

22. Liu ZL, Fu CW, Luan RS, Zhan SY, Chen WQ et al. (2008) The impact of complication on quality of life among diabetic patients in urban China. Chin J Epidemiol 29(10): 1029-1033.
23. Solli O, Stavem K, Sonbo Kristiansen I (2010) Health-related quality of life in diabetes: The associations of complications with EQ-5D scores. Health Qual Life Outcomes 8: 18.

24. Turner RC, Holman RR (1995) Lessons from UK prospective diabetes study.Diabetes Res Clin Pract (28 Suppl): S151-S157.

25. American Diabetes Association (2011) Summary of revisions to the clinical practice recommendations. (suppl 1): S3.

26. Rodbard HW, Jellinger PS, Davidson JA, Einhorn D, Garber AJ (2009) Statement by an American association of clinical endocrinologists/ American college of endocrinology consensus panel on type 2 diabetes mellitus. An algorithm for glycemic control. Endocr Pract 15(6): 54059.

27. Prospective Diabetes Study (UKPDS) Group (1998) Effect of intensive blood glucose control with metformin on complications in overweight patients with type 2 diabetes (UKPDS 34). Lancet, 352(9131): 854865.

28. Pinhas HO, Zeitler P (2005) The global spread of type 2 diabetes mellitus in children and adolescents. J Pediatr 146(5): 693-700.

29. Defronzo RA (2009) Banting Lecture. From the triumvirate to the ominous octet: a new paradigm for the treatment of type 2 diabetes mellitus. Diabetes 58(4): 773-795.

30. Fagot CA, Pettitt DJ, Engelgau MM, Burrows NR, Geiss LS, et al. (2000) Type 2 diabetes among North American children and adolescents: an epidemiologic review and a public health perspective.J Pediatr 136(5): 664-672.

31. Kahn SE, Hull RL, Utzschneider KM (2006) Mechanisms linking obesity to insulin resistance and type 2 diabetes. Nature 444(7121): 840-846.

32. Lyssenko V, Jonsson A, Almgren P, Pulizzi N, Isomaa B, et al. (2008) Clinical risk factors, DNA variants, and the development of type 2 diabetes. N Engl J Med 359(21): 2220-2232.

33. Ali MK, Echouffo TJ, Williamson DF (2012) How effective were lifestyle interventions in real-world settings that were modeled on the Diabetes Prevention Program? Health Aff 31(1): 67-75.

34. Katula JA, Vitolins MZ, Morgan TM, Lawlor MS, Blackwell CS, et al. (2013) The Healthy Living Partnerships to Prevent Diabetes study: 2 -year outcomes of a randomized controlled trial. Am J Prev Med 44(Suppl 4): S324-S332.

35. Knowler WC, Barrett CE, Fowler SE, Hamman RF, Lachin, et al. (2002) Reduction in the incidence of type 2 diabetes with lifestyle intervention or metformin. N Engl J Med 346(6): 393-403.

36. The community guide. (2015) Combined diet and physical activity promotion programs to prevent type 2 diabetes among people at increased risk. Guide to Community Preventive Services, USA.

37. Houle J, Beaulieu MD, Lussier MT, Del Grande, Pellerin JP, et al. (2012) Patients' experience of chronic illness care in a network of teaching settings. Can Fam Physician 58(12): 1366-1373.

38. Dabelea D, Bell RA, D'Agostino RB , Imperatore G, Johansen JM, et al. (2007) Writing Group for the SEARCH for in Youth Study Group. Incidence of diabetes in youth in the United States. JAMA 297(24): 2716-2724.

39. Haines L, Wan KC, Lynn R, Barrett TG, Shield JP (2007) Rising incidence of type 2 diabetes in children in the U.K. Diabetes Care 30(5): 10971101.

40. Funnel M (2006) The Diabetes Attitudes, Wishes, and Needs (DAWN) Study. Clin Diabetes 24(4): 154-155.

41. Nicolucci A, Burns Kovacs K, Holt RIG, Comaschi M, Hermanns N, et al. (2013) Diabetes Attitudes, Wishes and Needs second study (DAWN2 ${ }^{\mathrm{TM}}$ ): 


\section{Current Research in Diabetes \& Obesity Journal}

Cross-national benchmarking of diabetes-related psychosocial outcomes for people with diabetes. Diabet Med 30(7): 767-777.

42. International Alliance of Patients' Organizations (IAPO).(2012) Patient-Centred Healthcare Indicators Review.

43. Funnel M (2006) The Diabetes Attitudes, Wishes, and Needs (DAWN) Study. Clin Diabetes 24(4): 154-155.

44. Boyd CM, Reider L, Scharfstein D, Leff B, Wolff J, et al. (2010) The effects of guided care on the perceived quality of health care for multimorbid older persons: 18-month outcomes from a cluster-randomized controlled trial. J Gen Intern Med 25 (3): 235-242.

45. Glasgow RE, Wagner EH, Schaefer J, Mahoney LD, Reid RJ, et al. (2005) Development and validation of the Patient Assessment of Chronic Illness Care (PACIC). Med Care 43(5): 436-444.

46. Glasglow RE, Whitesides H, Nelson CC, King DK (2005) Use of the Patient Assessment of Chronic Illness Care (PACIC) with diabetic patients: relationship to patient characteristics, receipt of care, and self-management. Diabetes Care 28(11): 2655-2661.

47. McGuire BE, Morrison TG, Hermanns N, Skovlund S, Eldrup E, et al. (2010) Short-form measures of diabetes-related emotional distress the Problem Areas in Diabetes Scale (PAID)-5 and PAID-1. Diabetologia 53(1): 66-69.

48. Reddy J, Wilhelm K, Campbell L (2013) Putting PAID to diabetesrelated distress: the potential utility of the Problem Areas in Diabetes (PAID) scale in patients with diabetes. Psychosomatics 54(1): 44 - 51.

49. http://www.dawnstudy.com/

50. Hochberg G, Pucheu S, Kleinebreil L, Halimi S, Fructuoso V (2012) WHO-5, a tool focusing on psychological needs in patients with diabetes: The French contribution to the DAWN Study. Diabetes Metab 38(6): 512-522.
51. Frederick FT, Maharajh HD (2013) Prevalence of depression in type 2 diabetic patients in Trinidad and Tobago. West Indian med j 62(7): 628-631.

52. Gavard JA, Lustman PJ, Clouse RE (1993) Prevalence of depression in adults with diabetes. An epidemiological evaluation. Diabetes Care 16(8): 1167-1178.

53. Katon W, von Korff M, Ciechanowski P, Russo J, Lin E, et al. (2004) Behavioral and clinical factors associated with depression among individuals with diabetes. Diabetes Care 27(4): 914-920.

54. Lin EH, Katon W, Von Korff M, Rutter C, Simon GE, et al. (2004) Relationship of depression and diabetes self-care, medication adherence, and preventive care. Diabetes Care 27(9): 2154-2160.

55. Lustman PJ, Anderson RJ, Freedland KE, de Groot M, Carney RM, et al. (2000) Depression and poor glycemic control: a meta-analytic review of the literature. Diabetes Care 23(7): 934-942.

56. Black SA, Markides KS, Ray LA (2003) Depression predicts increased incidence of adverse health outcomes in older Mexican Americans with type 2 diabetes. Diabetes Care 26(10): 2822-2828.

57. Katon WJ, Rutter C, Simon G, Lin EH, Ludman E, et al. (2005) The association of comorbid depression with mortality in patients with type 2 diabetes. Diabetes Care 28(11): 2668-2672.

58. Carney C (1998) Diabetes mellitus and major depressive disorder: an overview of prevalence, complications, and treatment. Depress Anxiety 7(4): 149-157.

59. Lustman PJ, Griffith LS, Gavard JA, Clouse RE (1992) Depression in adults with diabetes.

60. Mahabir D, Gulliford MC (1997) Use of medicinal plants for diabetes in Trinidad and Tobago[Internet]. Pan Am J Public Health 1(3): 174-179.

Your next submission with Juniper Publishers
will reach you the below assets
- Quality Editorial service
- Swift Peer Review
- Reprints availability
- E-prints Service
- Manuscript Podcast for convenient understanding
- Global attainment for your research
- Manuscript accessibility in different formats
( Pdf, E-pub, Full Text, Audio)
- Unceasing customer service
Track the below URL for one-step submission
https://juniperpublishers.com/online-submission.php

